\title{
Integrating Observation Systems: an example from the Great Barrier Reef.
}

\author{
S.J. Bainbridge ${ }^{1}$, C.R. Steinberg ${ }^{1}$, M.L. Heron ${ }^{2}$ and M.J. Furnas ${ }^{1}$ \\ ${ }^{1}$ Australian Institute of Marine Science \\ Townsville, Queensland, Australia. \\ ${ }^{2}$ Marine Geophysical Laboratory, \\ James Cook University, \\ Townsville, Queensland, Australia.
}

\begin{abstract}
The Australian Integrated Marine Observing System (IMOS) project has deployed a set of focused observational equipment in the southern part of the Great Barrier Reef. This represents an good case study in what it actually means to deliver an integrated set of observations, how integration can be achieved and what the real benefits of true data integration are.
\end{abstract}

\section{INTRODUCTION}

There has been a commendable effort in recent years to establish long term Ocean Observing Systems in several countries e.g. IOOS in the USA, MYOCEAN in Europe, and IMOS in Australia, and these archives are laying a foundation for a productive research outcomes for global oceans. The Great Barrier Reef Ocean Observing System (GBROOS) is a regional node of the Australian Integrated Ocean Observing System (IMOS) [1] with focus points in the southern, central and northern sections of the Great Barrier Reef (GBR). The focus area in the southern part of the GBR is centred on Heron Island, within the Capricorn and Bunker Groups of islands and reefs. In this focus area a range of equipment has been deployed to fully understand the forcing functions and the impact of the Coral Sea on the reef systems of the Capricorn Bunkers. This area of focus will be used as a case study for integration within the deployed observing systems.

\section{COMPONENTS OF THE GBROOS PROJECT}

The GBROOS project consists of four components. At the largest scale is remote sensing data from a receiving station located at Townsville in north-east Australia. The data includes NOAA AVHRR data used for Sea Surface Temperature (SST) and MODIS Terra and Aqua data used for measures of ocean colour and productivity. Associated is a range of real-time validation data including a radiometer mounted on a commercial ferry, an optical reference station for ocean colour validation and underway systems on research vessels operating in Northern Australia.

At the next scale is an ocean surface HF radar installation in the southern GBR that provides real-time information on surface waves and currents. The installation covers an area of around 150 kilometres square at a resolution of four kilometre cells with data collected every ten minutes. The data are retrieved in real-time and can be processed into maps and plots showing surface current and waves, or time series at points of focus within the area.

The next level consists of an array of moorings along the GBR designed to monitor the flow of oceanic water into the reef matrix. The moorings are generally set up as pairs with one offshore deeper slope mooring paired with one inshore shelf mooring allowing the water column to be observed. Under the IMOS strategy, pairs of moorings are located in the northern, central and southern parts of the GBR. In the southern section moorings are located at $\mathrm{H} 1, \mathrm{H} 2$ and $\mathrm{H} 3$ as shown on Fig. 1, to the north, east and south of Heron Island, respectively. The moorings consist of sensors (temperature, salinity, temperature, pressure, dissolved oxygen, chlorophyll and turbidity) located in a profile down the mooring as well as a bottom mounted ADCP giving waves and currents.

The finest scale data comes from wireless sensor networks located at Heron and One Tree Islands. The sensor networks allow for intensive sampling of environments in shallow locations giving real-time information about water flows around individual corals as well as inflow and outflows from the reefs. Uniquely the sensor network uses smart controllers and high-speed two-way communications allowing the systems to be controlled and monitored in real time.

In the Capricorn - Bunker area the following components have been deployed as part of GBROOS and IMOS:

- A HF radar installation with transceivers located on Lady Elliot Island and Tannum Sands that cover the whole of the Capricorn - Bunkers area;

- Three deep water moorings (H1, H2 and H3) to the north, east and south of Heron Island, respectively;

- Sensor networks at Heron and One Tree Islands.

The location of the deployed equipment is shown in Fig One. 


\section{INTEGRATION}

Achieving real integration across the various components of GBROOS is not trivial and one often not fully understood when systems are designed and deployed. The GBROOS project is working to facilitate data integration from the sensor upwards through to data structures, metadata, data descriptions and data access mechanisms.

At the lowest level the project has standardised on a number of sensors and has looked to use well known 'traditional' oceanographic instruments that are robust and produce quality data. All sensors are calibrated and serviced in an equivalent manner. For the sensor network smart controllers are added to the oceanographic sensors so that real time data can be collected and centralised control is possible.

At the next level common data structures to hold all of the data have been built. Much of the data can be presented as simple X (latitude) / Y (longitude) / Z (depth) / T (time) / observation set of numbers, the ADCP spectra and the HF radar data are more complex but even they can be reduced (with processing) to a simple set of values. Underlying this is the need to set rigorous standards for these values. An example is depth; often equipment is deployed on floating buoys in which case the depth is relative to the surface or on anchored platforms in which case the depth is relative to the bottom. To be able to integrate data from a floating platform and a benthic platform requires you to be able to relate one to the other.

Part of data integration is a common set of processing and quality control steps. While there is no uniform representation for quality control across the marine community there are some examples of community based systems. GBROOS uses the IOC/IODE quality control flags [2] with a rules based software system to quality control the logged and real time data. The remote sensing and HF radar data are treated differently with the quality control for the remote sensing information coming from the processing software; in the case of the HF radars, the quality control protocols follow the IOC/IODE system.

The next level involves the description and representation of the data and the observing systems. The GBROOS project uses the ISO-19115 metadata standard [3] to describe the equipment and data with metadata records down to the sensor level. The metadata is hierarchical and so can be discovered using systems such as the IMOS web portal (see http://imos.aodn.org.au/webportal/).

The final level of integration is at the data access level. Using web services it is possible to make all of the data available to clients in a standardised manner. Client software can then pull data from the various web services and do the integration at that level. For the instrument data this can be done as a Sensor
Observation Service feed, for spectral and gridded data (such as the remote sensing and radar data) web services such as THREDDS (Thematic Real-time Environmental Distributed Data Services) [4] can be used to deliver the data. The client is able to fuse the data into a single data array that the user can then transform into truly integrated data products.

\section{ACHIEVING INTEGRATION}

For most projects integration is something that is bolted on after the project is designed and often after the equipment is deployed. To a degree this was the pattern with the GBROOS project. True integration requires the setting of standards and commonality at all stages from design to deployments to the management and dissemination of the data.

At the design level it is important that the equipment for the various components is at worst equivalent and at best identical. There needs to be confidence that the parameters being measured by each component have a similar level of accuracy and repeatability and that the maintenance of all instruments means that the stability of measurements through time is understood.

At the deployment level it is important that the readings taken by each component are in themselves equivalent. This means deploying sensors at similar depths with equivalent sampling times / frequencies and the processing done by the instrument is equivalent. Getting deployment level integration is often difficult as the environment will often dictate how the equipment is deployed and the differing environments of the open ocean and reef lagoons will dictate differing mounting, mooring and housing of the instruments. This is an area where planning is paramount in order to come up with a set of common deployment designs for each of the components and environments.

At the data level it is important that sampling frequencies are matched (such as every ten minutes or at least at even multiples of the fasted sampling), that instrument clocks are synchronised and checked, that the same time datum is used (e.g. UTC and not local time) and that units and processing levels are matched.

The higher level data integration is more straightforward and relies on the data being stored in the one system or in a set of systems that can be queried as if they were one system. This may require consistent use of identifiers, common higher level data structures (such as site, equipment and identifier tables) and a set of standard queries that is able to pull data from a set of tables or repositories.

The final level of integration is at the user level. This means presenting the user with a set of data that 'looks' the same. By this we mean it has the same quality control identifiers / processing, the data are in equivalent units in terms of both time and the value being measured, parameters such as location, time and depth are in the same datum as well as units and which, when co-plotted, neatly overlay with no additional processing. 
So how to achieve integration? The first step is to actually define what is meant by integration given the components of the observing system that are to be deployed and the questions or issues that it is to address. For most systems it will mean that data from one component of the observing system can easily and logically be overlayed with other data to form a seamless new data product that is both valid and which has scientific meaning.

The second step is to set standards for everything and never deviate from these unless the consequences of this is clearly understood. All standards should reflect those in use by the community with the caveat that the standards used should make sense to the end user. One example of this is the measurement units where often the users will be more familiar with older units (such as knots for wind speed) that the correct SI units such as metres per second.

The next step is to build data systems to ingest all of the data being collected and work to build linkages between the systems so that they can appear as a single system even if they are made up of quiet separate components. For example it is now possible to build data systems that include relational databases, flat files and XML files and run single queries over the collection of components as if it was a single system. The data systems should include the quality control processing and checking, any pre-processing of data, metadata and other data descriptions such as SensorML.

The final step is to document everything! Only by ensuring that everything is recorded, preferably in the metadata, can a user decide if the data can logically and validly be merged.

\section{CYClONE HAMish}

In March 2009 tropical cyclone Hamish traversed down the GBR and through the Capricorn - Bunkers area missing Heron and One Tree Islands by a mere 130 kilometres while being rated as a Category-4 cyclone. The GBROOS infrastructure recorded above water meteorological parameters at Heron and One Tree Island, in water parameters from the three deep water moorings and wave heights and currents from the HF radar.

The initial damage analysis using wind strength based models [5] indicated marginal damage should have been sustained. Follow up visual surveys currently underway indicate large scale damage, analysis of the observation data shows large sea and swell heights and so indicates that the wind based model of impact did not represent what actually occurred with cyclone Hamish.

By having a range of integrated observing infrastructure deployed it becomes possible to better understand the impacts of events such as cyclones and, through improved models and understanding, to better predict what the potential impact of future storms will be. Reliance on just wind measurements alone would not have explained the level of damage sustained, only through the combination of data from the observing systems can the documented level of damage be explained.

\section{WHAT INTEGRATION DELIVERS}

The ultimate goal of the integration exercise in the southern GBR area was to be able to track the impact of large scale oceanic events across a range of scales from the tens of kilometres that ocean basins represent down to tens of centimetres at the scale of an individual coral head. In this way we can understand how the large scale processes interact and force the smaller scale patterns that ultimately impact the organisms of the GBR. It also allows us to separate processes occurring at differing scales and so it becomes possible to discern between ocean waves and lagoonal tidal driven waves.

\section{WHY INTEGRATION MATTERS}

Integration matters because in the area of climate and change new understandings will come more and more from multi-dimensional data sets. It is the combination of data, from a range of sources, that is essential to understanding the processes and outcomes from a changing world. Integration is the key to delivering this new generation of data products, if the data components are not designed with integration in mind then it becomes difficult or impossible to extract the knowledge that may lie within. Simple issues such as differing deployment depths can make it hard to impossible to fuse data that, apart from that one dimension, is amenable to integration.

The other reason is that the data will almost defiantly be used for very different purposes than what it was collected for. The re-use and re-analysis of data will become a more critical way of extracting understanding in a world where it is often impossible to go back and sample an environment because it either doesn't exist anymore or if it does it has been altered.

\section{CONCLUSION}

While many systems use the term 'integrated' there is often very little actual design work to ensure that true data integration is possible. Standards in equipment, deployment practices, data structures, processing and quality control and in data access are all required to enable data to be truly integrated in a meaningful way. This paper details the work that the GBROOS project has done to facilitate integration at all levels; from sensor and deployment design to data representations, data descriptions and data access.

\section{ACKNOWLEDGMENT}

The GBROOS project is part of the Australian Integrated Marine Observing System (IMOS) project and is funded by the Queensland State Government and the Australian Federal Government through the National Collaborative Research Infrastructure Strategy (NCRIS). 


\section{REFERENCES}

[1] IMOS 2010. Australia's Integrated Marine Observing System http://www.imos.org.au

[2] UNESCO/IOC/IODE \& MAST, 1993. Manual of Quality Control Procedures for validation of Oceanographic Data - UNESCO Manuals and Guides 26

[3] ISO, 2003. Geographic Information metadata. http://www.iso.org/iso/iso_catalogue/catalogue_tc/catalogue_detail.htm? csnumber $=26020$
[4] Unidata 2010. Thematic Real-time Environmental Distributed Data Service http://www.unidata.ucar.edu/projects/THREDDS/

[5] Puotinen ML 2007 Modelling the risk of cyclone wave damage to cora reefs using GIS: A case study of the Great Barrier Reef, 1969-2003. Int. J. Geogr. Inf. Sci. 21: 97-120

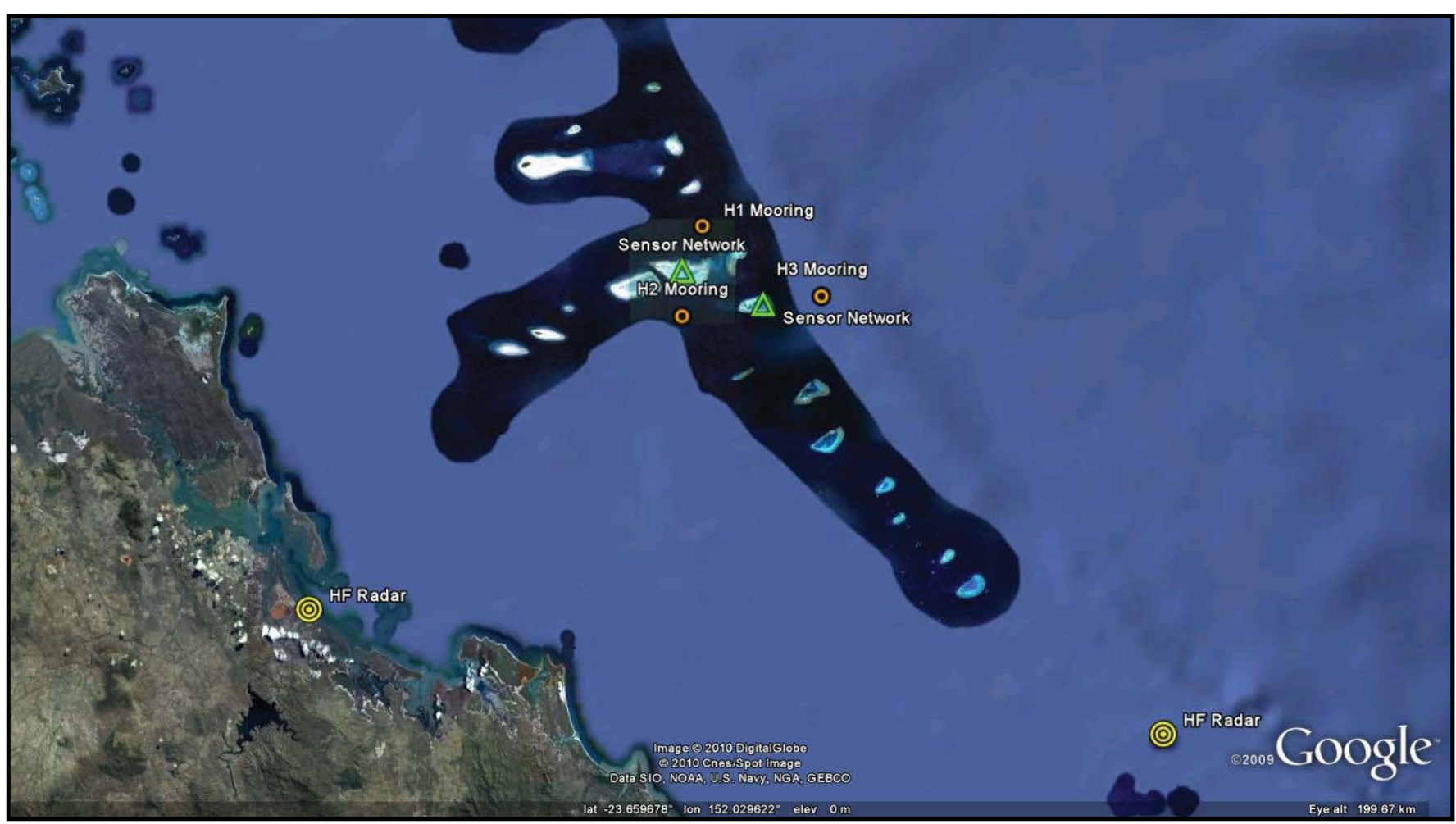

Figure 1. Location of deployed equipment in the southern Great Barrier Reef. Yellow are circles are HF radar transceivers, green triangles are on-reef sensor networks and orange dots are deep water ocean moorings. Image copyright Google 2010. 